\title{
Energy Efficiency Analysis for Dynamic Routing in Optical Transport Networks
}

\author{
Vizcaíno, Jorge López; Ye, Yabin; Tafur Monroy, Idelfonso
}

Published in:

2012 IEEE International Conference on Communications (ICC)

Link to article, DOI:

$10.1109 /$ ICC.2012.6363876

Publication date:

2012

Document Version

Early version, also known as pre-print

Link back to DTU Orbit

Citation (APA):

Vizcaíno, J. L., Ye, Y., \& Tafur Monroy, I. (2012). Energy Efficiency Analysis for Dynamic Routing in Optical Transport Networks. In 2012 IEEE International Conference on Communications (ICC) (pp. 3009-3014 ). IEEE. I E E E International Conference on Communications https://doi.org/10.1109//CC.2012.6363876

\section{General rights}

Copyright and moral rights for the publications made accessible in the public portal are retained by the authors and/or other copyright owners and it is a condition of accessing publications that users recognise and abide by the legal requirements associated with these rights.

- Users may download and print one copy of any publication from the public portal for the purpose of private study or research.

- You may not further distribute the material or use it for any profit-making activity or commercial gain

- You may freely distribute the URL identifying the publication in the public portal 


\title{
Energy Efficiency Analysis for Dynamic Routing in Optical Transport Networks
}

\author{
Jorge López Vizcaíno ${ }^{1,2}$, Yabin Ye ${ }^{1}$, Member, IEEE, and Idelfonso Tafur Monroy ${ }^{2}$, Member, IEEE \\ ${ }^{1}$ Huawei Technologies Duesseldorf GmbH, Riesstr. 25, 80992 Munich, Germany \\ ${ }^{2}$ Department of Photonics Engineering, Technical University of Denmark, Ørsteds Plads, B. 343, 2800 Kgs. Lyngby, Denmark \\ jorge.vizcaino@ huawei.com
}

\begin{abstract}
The energy efficiency in telecommunication networks is gaining more relevance as the Internet traffic is growing. The introduction of OFDM and dynamic operation opens new horizons in the operation of optical networks, improving the network flexibility and its efficiency. In this paper, we compare the performance in terms of energy efficiency of a flexible-grid OFDM-based solution with a fixed-grid WDM network in a dynamic scenario with time-varying connections. We highlight the benefits that the bandwidth elasticity and the flexibility of selecting different modulation formats can offer compared to the rigidity of the conventional networks.
\end{abstract}

Keywords; Elastic optical path network; energy efficiency; dynamic routing; optical OFDM; Routing, Modulation Level and Spectrum Allocation

\section{INTRODUCTION}

In the following years, the Internet traffic is expected to grow even more dramatically than previously, since according to [1], in 2015 the global IP (Internet Protocol) traffic is predicted to be four times larger than that in 2010. This growth is justified not only by the increase in the number of users and devices connected to the Internet, but also by the high bandwidth requirements of some popular applications such as Internet video, which at the end of 2012 is forecasted to account for more than the half of the global Internet traffic. Accordingly, the telecom operators and industry have mainly focused the research on increasing the network capacity, which is usually accompanied by an increase in energy consumption. In 2009, according to [2], the power consumption of the ICT (Information and Communications Technology) already accounted for the $4 \%$ of the global energy consumption. However, it is expected that the forecasted traffic growth will make the energy consumption to become a significant problem for the operators, having not only economical (affecting capital and operational expenditures), but also ecological implications, since the ICT sector is responsible for the production of the 2$2.5 \%$ of the global greenhouse emissions, as estimated by the ITU (International Telecommunication Union) in [3]. In this manner, the energy efficiency is becoming one of the key design parameters for the network planning and its operation.

The current optical transport technologies are based on WDM (Wavelength Division Multiplexing), in which the wavelengths are assigned in a permanent manner. This assignment, in a single-layer design, presents two inefficiency problems: the first one due to the coarse granularity of a wavelength, as the actual demand could be lower than the assigned wavelength capacity; and the second one due to the static assignment, since a wavelength is permanently assigned without considering the traffic variations that might occur in time. These inefficiencies are to become more significant in the near future with the evolution in wavelength capacity of WDM networks. Therefore, the network's efficiency can be improved by making the network more flexible and dynamic, i.e. by introducing a finer granularity and by considering the time fluctuations in the demands. In order to provide a finer granularity, optical OFDM (Orthogonal Frequency Division Multiplexing), combined with coherent detection may play an important role to substitute the conventional WDM networks. This modulation technique offers two dimensions of flexibility to better adjust the transmission rate to the actual demand: the elastic bandwidth transmission by selecting a variable number of subcarriers, and the possibility of using different modulation formats for subcarriers. This concept is referred in the literature as Spectrum-sLICed Elastic optical path network "SLICE" [4].

In our previous work [5], the benefits of an Elastic OFDMbased network in terms of energy efficiency as a solution for the network planning problem (offline routing) were investigated. This analysis has now been extended to a dynamic scenario considering time-varying connections that arrive randomly to the network and remain active for a variable and limited period of time. Accordingly, in this paper, an Elastic OFDM-based network and the conventional fixed-grid WDM networks, both with a Single Line Rate (SLR) and with Mixed Line Rate (MLR), are compared in terms of energy efficiency and network blocking. Even though, the introduction of the Elastic OFDM-based network is a topic under extensive research, there are only few reported results studying a dynamic scenario, and most of them focus on the blocking probability analysis, such as [6] which shows a superior performance of the grid-less approach compared to the conventional fixed-grid one. To the best of authors' knowledge, this is the first analysis of a dynamic scenario for an Elastic OFDM-based network in terms of energy efficiency.

The paper is organized as follows. Section II presents some considerations for the resource allocation in the different networks. Section III introduces the values of power consumption of the network elements. Section IV explains the dynamic routing heuristic algorithms. Section V presents the simulation results, and finally Section VI concludes the paper.

\section{NETWORK CONSIDERATIONS}

\section{A. Elastic OFDM Network}

A frequency slot (minimum bandwidth unit for a subcarrier) of $12.5 \mathrm{GHz}$ has been adopted; hence the C-Band (4 $\mathrm{THz}$ ) is divided into 320 frequency slots. Besides, in order to maintain the orthogonality condition among the subcarriers, the subcarrier spacing must be equal to the baud rate. Thus, the 
transmission rate for a single subcarrier can be 12.5, 25, 37.5, 50, 62.5 and $75 \mathrm{~Gb} / \mathrm{s}$ for BPSK, QPSK, 8QAM, 16QAM, 32QAM and 64QAM respectively. Then, in order to provide different transmission rates, the CO-OFDM (Coherent Optical OFDM) transponder can combine a number of contiguous subcarriers, modulated with the same format, into a lightpath (or optical path). In addition to the subcarriers used for data transmission, a guard band composed of two subcarriers (25 $\mathrm{GHz}$ ) is introduced in between the adjacent optical paths, allowing the bandwidth variable OXC (Optical Cross Connect) to add or drop any of the paths. In the selection of the subcarrier modulation format, the transmission reach of the modulation format must be considered. Based on the values estimated in [7], transmission reaches of 4000, 2000, 1000, 500, 250 and 125 kilometers have been assumed for BPSK, QPSK, 8QAM, 16QAM, 32QAM and 64QAM respectively.

\section{B. WDM Networks}

In the WDM network, a fixed-grid of $50 \mathrm{GHz}$ channels (80 wavelengths in the C-band) has been adopted, considering three different line rates of 10,40 and $100 \mathrm{~Gb} / \mathrm{s}$, with transmission reaches of 3000, 1500 and $1200 \mathrm{~km}$ respectively. Two different network approaches have been analyzed: one with a single transmission rate (SLR), and another one combining the three mentioned line rates (MLR). In this latter approach, since different technologies are mixed, the cross-talk effect might affect the signal quality and the transmission reach. In order to minimize this effect, the C-band has been divided into two different wavebands, separated by a guard band of $200 \mathrm{GHz}$ : the first one for $10 \mathrm{~Gb} / \mathrm{s}$, and the second one for both 40 and $100 \mathrm{~Gb} / \mathrm{s}$ connections, that can be alternated without considerably affecting the signal quality of each other.

\section{POWER CONSUMPTION}

\section{A. Transponders}

In the WDM network case, the values of 34, 98 and $188 \mathrm{~W}$ in [8] have been considered for the transponders of 10, 40 and $100 \mathrm{~Gb} / \mathrm{s}$ respectively. In the Elastic OFDM-based network, due to the commercial unavailability of a bandwidth variable transponder (CO-OFDM transponder), some assumptions have been made, as detailed in [5], to derive the values of power consumption. Thus, the power consumption of a single polarization CO-OFDM transponder can be interpolated as function of its transmission rate $(T R)$ as in (1). Accordingly, the power consumption of the CO-OFDM transponder, considering a single subcarrier, for the different modulation formats is presented in Table I. Furthermore, an additional $20 \%$ of power consumption is considered for the overhead contribution at the transponders for both types of networks.

$$
P C(W)=1.25 \cdot T R(G b / s)+31.5
$$

\section{B. Optical Cross Connects (OXC)}

The power consumption of a fixed bandwidth OXC (WDM) and a bandwidth variable OXC (Elastic OFDM) have been assumed to be similar and dependent on the node degree $N$, as estimated in [8]. Furthermore, an overhead contribution of $150 \mathrm{~W}$ per node location has been assumed as follows:

$$
\operatorname{PCOXC}(W)=N \cdot 85+150
$$

TABLE I. POWER CONSUMPTION OF A CO-OFDM TRANSPONDER FOR DIFFERENT MODULATION FORMATS

\begin{tabular}{|c|c|c|}
\hline Mod. Format & $\begin{array}{c}\text { Subcarrier } \\
\text { Capacity }(\mathbf{G b} / \mathbf{s})\end{array}$ & $\begin{array}{c}\text { Power Consumption } \\
(\mathbf{W})\end{array}$ \\
\hline BPSK & 12.5 & 47.13 \\
\hline QPSK & 25 & 62.75 \\
\hline 8QAM & 37.5 & 78.38 \\
\hline 16QAM & 50 & 94 \\
\hline 32QAM & 62.5 & 109.63 \\
\hline 64QAM & 75 & 125.23 \\
\hline
\end{tabular}

\section{Optical Line Amplifiers}

The value in [8], considering $60 \mathrm{~W}$ per fiber pair has been considered for an EDFA (Erbium Doped Fiber Amplifier) in both types of networks. Moreover, 140 additional watts per amplifier location are considered as overhead contribution.

\section{DYNAMIC ROUTING ALGORITHMS}

\section{A. Dynamic Scenario Parameters}

Unlike the static scenario, in which a traffic demand matrix with the fixed demands between nodes is known before the provisioning of the resources; in a dynamic scenario, in order to emulate the traffic, the demands have been assumed to arrive in the network according to a Poisson distribution with a mean arrival rate $\lambda$ (mean number of connection requests/time unit), and the holding time follows an exponential distribution with intensity $\mu$ (mean number of finished connections/time unit). In order to analyze the performance under different traffic load conditions, the simulations have been performed for different values of offered traffic $(\lambda / \mu)$. The network traffic matrix of the static scenario [10] is used to specify the node connectivity (i.e. which network nodes exchange information) and the maximum demand value between two nodes. Moreover, to emulate the dynamic network operation, the source and destination nodes for a new flow are randomly selected from the possible sourcedestination pairs in the traffic matrix with a transmission rate that fluctuates uniformly in an interval from the $1 \%$ to the 100 $\%$ of the maximum traffic demand value.

\section{B. Resource Allocation Algorithms}

The resource allocation problems are referred as RWA (Routing and Wavelength Assignment) and RMLSA (Routing, Modulation Level, and Spectrum Allocation) for the WDM and the Elastic OFDM-based networks respectively. The main differences of the RMLSA lie in the contiguous spectrum allocation constraint of OFDM, the different modulation format possibilities, and the higher number of subcarriers (320) compared to the wavelengths in WDM (80). Since these problems are NP-complete, different dynamic routing heuristic algorithms are proposed, which consider each demand one-byone in order of its arrival time. The time-varying demands are referred as flows; whereas a lightpath refers to the all-opticalpath that is established between two network nodes provided that enough and common resources (wavelengths or subcarriers) are available in all the links of the path, being able to convey one or several flows. The input parameters are: the traffic matrix, the physical topology, the power consumption values, the transmission reach of each modulation format, and the offered traffic. This section presents the operation of these algorithms for the different events that happen in the network. 


\section{1) RMLSA in Elastic OFDM network}

\section{a) New Flow Request (no existing lightpath)}

If a new flow request arrives and there is no active communication between the source and destination nodes, the establishment of a new lightpath for the possible combinations of path and modulation format is evaluated (Fig. 1), searching for a common block of contiguous subcarriers in the links of the path following the First Fit Packing algorithm. The size of this block of subcarriers $(N o S u b c)$ is determined by the number of data subcarriers NoDataSubc (demand/capacity single subcarrier), plus the two guard band subcarriers. Finally, if the allocation is possible (available common subcarriers in all the links of the path), a metric based on the end-to-end lightpath power consumption (MetricPC) is calculated considering the power consumption of the transponders (PCT), and an approximate contribution from the EDFAs and OXCs along the path (PCLinks). PCT is the product of the number of data subcarriers (NoDataSubc) and the power consumption of a single subcarrier for the corresponding modulation format $(P C S u b c)$ from Table I, as in (3). On the other hand, PCLinks is calculated based on the proportion of resources that the lightpath would occupy in the links (number of subcarriers of the lighpath $N o S u b c$ with respect to the total number of subcarriers in a fiber TotalNoSubc), and the addition of the power consumption of the EDFAs (PCEDFAs) and OXCs $(P C O X C s)$ in the path, as in (4).

$$
\begin{gathered}
P C T=\text { NoDataSubc } \times \text { PCSubc } \\
\text { PCLinks }=\frac{\text { NoSubc }}{\text { TotalNoSubc }}(\text { PCEDFAs }+ \text { PCOXCs }) \\
\text { MetricPC }=\text { PCT }+ \text { PCLinks }
\end{gathered}
$$

Once MetricPC is obtained in (5), it is compared with the lowest metric calculated previously (LowestMetricPC), and in case of being lower, the resource allocation information (link sequence, modulation format, frequency slots, power consumption) is stored in the MostEfficientAllocation. Thus, after evaluating all the combinations, the most efficient one can be selected for the spectrum allocation.

\section{b) New Flow Request (active lightpath)}

If there is an active communication between source and destination nodes, different possibilities are evaluated to reuse the resources of the active lightpath (Fig. 2). If grooming the demand into the existing lightpath is not possible, a lightpath capacity upgrade (expanding bandwidth or increasing modulation order) is checked, selecting the most energy efficient solution in case of several options. Otherwise, the establishment of a new lightpath is evaluated.

\section{c) Flow termination}

Once a flow terminates, if there are no more flows sharing the same lightpath, the allocated resources are released. Otherwise, it is checked whether a release of resources (reducing the number of subcarriers), or a decrease in the modulation order is more advantageous in terms of power consumption. Furthermore, the data that were transmitted and the energy consumption of the transponders in the duration of the flow are calculated, and will be used to compute the final

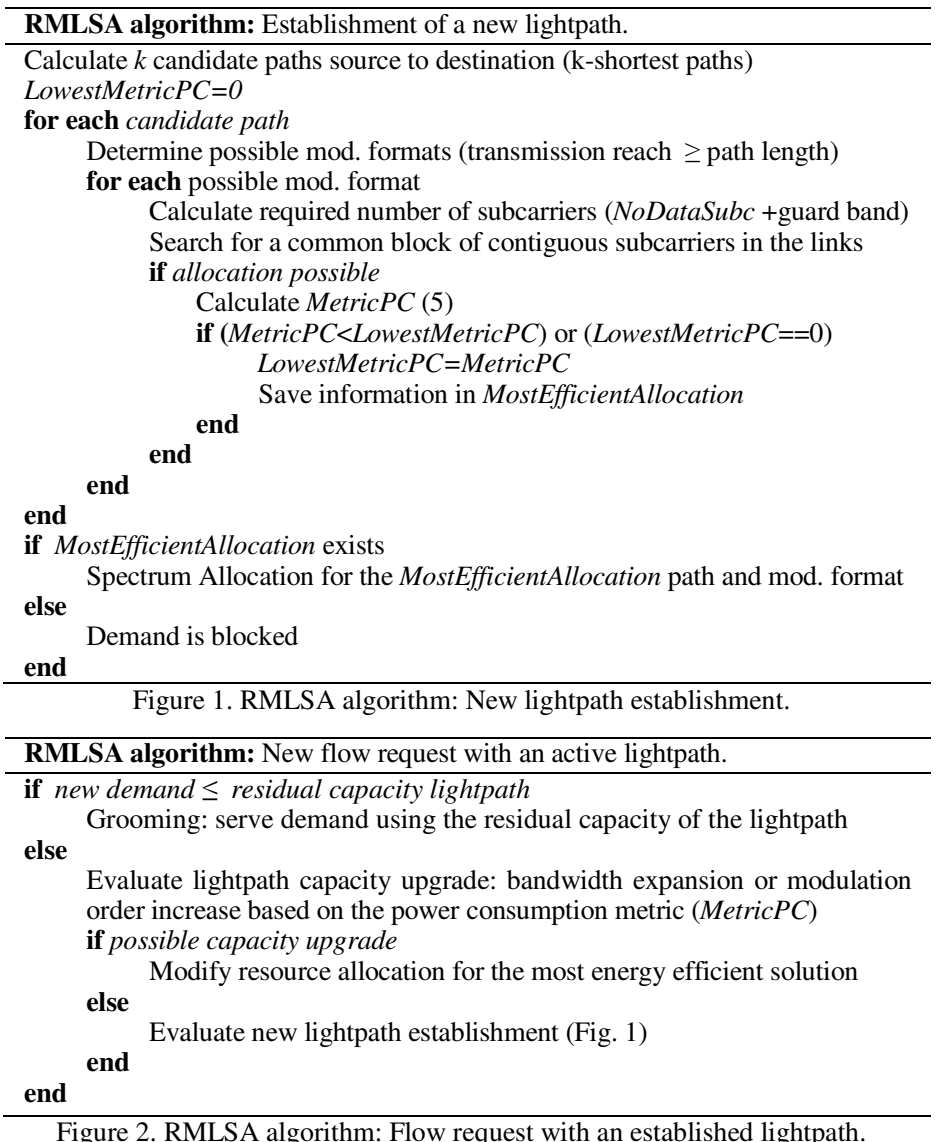

measures. The data transmitted is given by the product of the transmission rate of the flow (TRFlow) and the flow duration (6). On the other hand, the energy consumption due to the flow transmission is obtained by multiplying the power consumption of the transponder with the duration of the flow (7).

DataTransmitted $($ bits $)=$ TRFlow $($ bit $/ s) \cdot$ FlowDuration $(s)(6)$

$$
\operatorname{ECFlow}(W \cdot s)=P C T(W) \cdot F l o w D \text { uration }(s)
$$

\section{2) RWA in WDM SLR network}

\section{a) New Flow Request(no existing lightpath)}

The procedure is simpler than that in the RMLSA due to the presence of a single line rate, and the possible assignment of wavelength without the contiguous spectrum allocation constraint. As presented in Fig. 3, the allocation is evaluated for all the candidate paths, selecting the least power consuming path according to (5), but considering (8) and (9) instead of (3) and (4) respectively:

$$
\begin{aligned}
& P C T=N o \lambda s \times P C W D M T r a n s p o n d e r \\
& \text { PCLinks }=\frac{\text { Noגs }}{\text { TotalNoגs }}(P C E D F A s+\text { PCOXCs })
\end{aligned}
$$

\section{b) New Flow Request (active lightpath)}

The possibilities of either grooming the demand using the residual capacity of the lightpath, or assigning additional wavelengths along the same path are firstly checked. Otherwise the establishment of a new lightpath is evaluated as in Fig.3. 


\section{c) Flow termination}

Once a flow terminates, it is verified whether it implies the release of the assigned wavelengths. Moreover, the energy consumption, and transmitted data in the duration of the flow are calculated as in the RMLSA algorithm.

\section{3) RWA in WDM MLR network}

\section{a) New Flow Request(no existing lightpath)}

As shown in Fig.4, the assignment is similar to the RWA for SLR, but considering the two different bands. Thus, in the first waveband $(10 \mathrm{~Gb} / \mathrm{s})$ the assignment follows the First Fit packing algorithm starting from the first wavelength, whereas in the second band (40 and $100 \mathrm{~Gb} / \mathrm{s}$ ) it starts from the last one. This allows for a possible movement of the guard band increasing the wavelengths in a band. For each demand, first the $k$ shortest paths are calculated, and then all the possible combinations of line rates based on the shortest path are listed. Then, the list is sorted in ascending order based on power consumption. For instance, for a demand of $85 \mathrm{~Gb} / \mathrm{s}$ with the shortest path length of $1300 \mathrm{~km}$, the list of possible line rate combinations would be: $2 \times 40 \mathrm{~Gb} / \mathrm{s}+1 \times 10 \mathrm{~Gb} / \mathrm{s} ; 3 \times 40 \mathrm{~Gb} / \mathrm{s}$; $1 \times 40 \mathrm{~Gb} / \mathrm{s}+5 \times 10 \mathrm{~Gb} / \mathrm{s} ; 9 \times 10 \mathrm{~Gb} / \mathrm{s}$. From the first possible line rate combination, for each of the candidate paths, the allocation is firstly evaluated without moving the existing guard band. If the allocation is not possible in a path, a movement of the guard band in the links can be considered to increase the number of wavelengths in a band. After all the $k$ paths have been checked, if a feasible solution cannot be obtained, the following line rate combinations are checked sequentially. More details about the algorithm can be found in [9].

\section{b) New Flow Request (active lightpath)}

Similar steps to those of the RWA algorithm for SLR.

\section{c) Flow termination}

Similar steps to those of the RWA algorithm for SLR.

\section{Final measures}

The total energy consumption of the network is calculated by adding the total energy consumption at the transponders, the EDFAs and the OXCs. The contribution from the transponders is obtained by the addition of the energy consumed for each of the flows (7); whereas the one from the EDFAs and OXC are obtained by the total energy consumed by these network elements in the total simulated time (assumed to be always $\mathrm{ON})$. The total data transmitted is obtained by summing the data successfully transmitted in the different flows (6). Then the energy efficiency of the network (bits/Joule) is defined as the ratio between the total data transmitted, and the total energy consumed in the network (10). Besides, the network blocking has also to be considered, as it determines the number of resources that the network requires to operate. The system is assumed to be a loss system, so a rejected connection request is lost without any further re-attempt. Since demands with higher transmission rate $(T R)$ are more likely to be blocked, this fact is considered in the blocking ratio measure (11). The first set of attempts is dropped from the calculation as the network is not in steady state at the beginning of the simulation.

$$
\text { EnEff }(\text { bits } / \text { Joule })=\frac{\text { TotalDataTransmitted }(\text { bits })}{\text { TotalEnergyConsumption }(W \cdot s)}(10)
$$

RWA SLR algorithm: Establishment of a new lightpath.

Calculate $k$ candidate paths source to destination (k-shortest paths)

Calculate the required number of wavelengths (demand/wavelength line rate) LowestMetric $P C=0$

for each candidate path

Determine transparent reach possibility (transmission reach $\geq$ path length) if transparent reach

Search for common wavelengths in the links of the path if allocation possible Calculate MetricPC (5) if $($ Metric $P C<$ LowestMetric $P C)$ or $($ LowestMetric $P C==0)$ LowestMetric $P C=$ Metric $P C$ end Save information in MostEfficientAllocation

end end

end

if MostEfficientAllocation exists else

Wavelength Assignment for the path in MostEfficientAllocation

Demand is blocked end

Figure 3. RWA SLR algorithm: New lightpath establishment.

RWA MLR algorithm: Establishment of a new lightpath.

\section{LowestMetric $P C=0$}

Calculate $k$ candidate paths source to destination (k-shortest paths)

List all the possible line rates combinations based on the shortest path (transmission reach $\geq$ path length)

Sort the line rates combinations list in ascending order based on power consumption

while list of line rate combination is not empty

for each candidate path

if transparent reach (for the considered line rate combination) if $10 G$ in line rate combination

Search for common wavelengths in the $1^{\text {st }}$ band of the links

if allocation not possible

Search for common wavelengths by moving the guard

band to the right direction end

end

if $40 G / 100 G$ in line rate combination

Search for common wavelengths in the $2^{\text {nd }}$ band of the links if allocation not possible

Search for common wavelengths by moving the guard

band to the left direction

end

end

if allocation possible

Calculate MetricPC (5)

if $($ MetricPC $<$ LowestMetricPC) or (LowestMetricPC $==0)$ LowestMetric $P C=$ Metric $P C$

end

Save information in MostEfficientAllocation

end

end

end

if LowestMetricPC !=0

Break

else

Remove the considered line rate combination from the list end

end

if MostEfficientAllocation exists

Wavelength Assignment for the selected path and line rates

else

Demand is blocked

end

Figure 4. RWA MLR algorithm: New lightpath establishment.

Service Blocking Ratio $=\frac{\sum \text { TR.BlockedDemands }}{\sum \text { TR.TotalDemands }}$ 


\section{SimUlation RESUlTS}

In order to check the influence of the network size, the simulations were performed for both a long-haul and an ultra long-haul networks. It is important to note that a single fiber pair has been considered per link.

\section{A. DT Network}

The DT (Deutsche Telekom) network is a long-haul country-sized network (diameter of $800 \mathrm{~km}$ ) composed of 14 nodes and 23 bidirectional links, as presented in [10]. It is a fully-transparent network, given that all the demands between the network nodes can be served without any regeneration. The traffic demand matrix from [10] has been adopted as a reference, with 182 possible source-destination pairs, and scaled up by 30 times (average flow demand of $230.03 \mathrm{~Gb} / \mathrm{s}$ ). In the algorithms, five shortest paths $(k=5)$ were used as candidates. Each simulation, regardless of the offered traffic, considered 40,000 connection requests, and the system to be in steady state after the first 4,000 requests. The simulations were performed for values of offered traffic $(\lambda / \mu)$ from 10 to 800 .

The results in Fig. 5 show, as a common tendency for different networks, an increase in energy efficiency with the offered traffic. The reason is that, despite a similar amount of data is requested to be transmitted (same number of events and same average demand), a higher traffic load implies a reduction in the power consumption contribution of the EDFAs and OXCs, which are assumed to be always turned on. Moreover, the energy efficiency of the transponders increases with the offered traffic, since the number of simultaneous flows sharing source and destination nodes also grows, allowing for the grooming of several flows into the same lightpath.

As Fig. 5 shows, the MLR and the Elastic OFDM approach present similar energy efficiency for any value of offered traffic, and outperforming the SLR systems. In low traffic load conditions, the benefit comes from its finer granularity, but as the traffic load increases, the granularity becomes less relevant, and the total network capacity gains more importance. Thus, due to its higher network capacity, the $100 \mathrm{~Gb} / \mathrm{s}$ network improves its energy efficiency as the traffic load increases, even reaching similar performance to the MLR and the Elastic OFDM network. Regarding the blocking ratio, it becomes considerably high for the 10 and $40 \mathrm{~Gb} / \mathrm{s}$ networks as the traffic load increases, as shown in Fig. 6, since the network is not able to handle more traffic. On the other hand, the other three networks present a better result in blocking due to their higher network capacity. Among these three networks, the Elastic OFDM offers a considerably better performance than the $100 \mathrm{~Gb} / \mathrm{s}$ and the MLR approaches, which is justified by the improvement in spectral efficiency due to its grid-less operation and the use of high spectral efficient modulation formats. Concerning the other two networks, the MLR is slightly more affected by blocking than the $100 \mathrm{~Gb} / \mathrm{s}$, due to the presence of the guard band that eventually reduces its useful network capacity. Based on the results, in energy efficiency and service blocking ratio, the Elastic OFDM network is the most energy efficient solution, offering not only the best results in energy efficiency under any traffic condition, but also the ability to handle more traffic, with the consequent savings in cost and energy consumption.

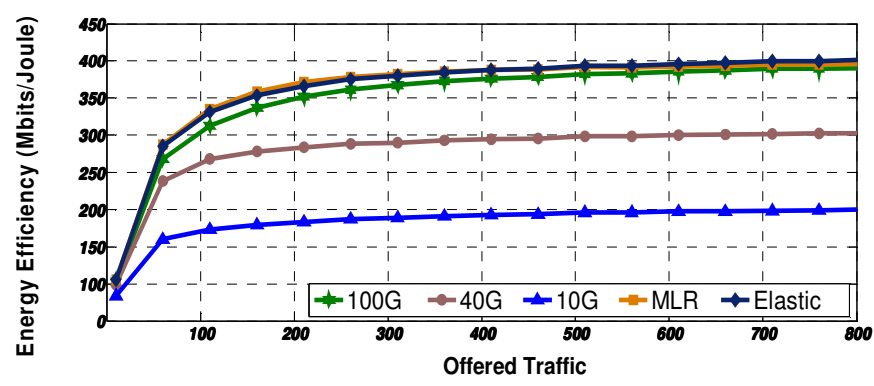

Figure 5. DT Network: Energy Efficiency.

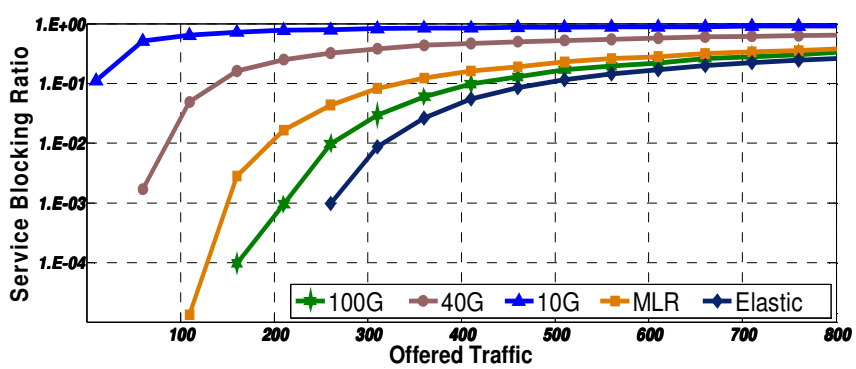

Figure 6. DT Network: Service Blocking Ratio.

\section{B. GÉANT2 Network}

GÉANT2 is the European research and education network with a continental size (diameter of $7000 \mathrm{~km}$ ), composed of 34 nodes and 52 bi-directional links as shown in [10]. Its large dimension makes the transparent reach not possible for some connections due to the accumulation of physical impairments along the path, so regenerators are necessary in some network nodes to recover the signal quality. Since regenerators are highly energy-consuming, simulations were performed to determine the placement of a minimum number allowing for the realization of all the connections in the traffic matrix. As a result, regenerators with wavelength conversion were placed in four network nodes (Switzerland, Denmark, Luxembourg, and Malta). Thus, a demand between nodes separated by a long distance could be served by several consecutive lightpaths. The traffic demand matrix in [10] with 784 possible sourcedestination pairs, has been adopted as reference, and scaled up by 20 times (average demand of $32.60 \mathrm{~Gb} / \mathrm{s}$ ). In the algorithms, twelve shortest-path $(k=12)$ were used as candidates. Each simulation, regardless of the offered traffic, considered 60,000 connection requests, and the system to be in steady state after the first 6,000 requests. The simulations were performed for values of offered traffic $(\lambda / \mu)$ ranging from 10 to 800 . In this network, due to long paths lengths of some connections, only the $10 \mathrm{~Gb} / \mathrm{s}$ has been considered for a SLR network, since the 40 and $100 \mathrm{~Gb} / \mathrm{s}$ would imply a high number of regenerations, with the consequent increase in cost and energy consumption.

The network topology and the demand value play an important role in the network energy efficiency, as it can be observed from the significant difference in the values of energy efficiency obtained for this network (Fig. 7) and the ones for the DT Network (Fig. 5). The lower energy efficiency in the GÉANT2 is justified by the considerably higher power consumption of EDFAs and OXCs, $99280 \mathrm{~W}$, compared to that in the DT Network, 14010 W. Thus, due to the low average demand, this contribution clearly dominates the total power 
consumption under low traffic conditions. This influence is shown in Fig. 7, where similar energy efficiency is presented by the three networks. However, as the traffic load increases, a difference in performance among the three networks is observed. The reason is that the spectrum occupancy increases with the traffic load, so that for high values, the maximum network capacity becomes the most relevant factor, determining the amount of traffic that the network is able to handle, and affecting therefore the energy efficiency. The network capacity can be extracted from the blocking ratio measure of Fig. 8. Thus, it can be observed the considerable high blocking presented by the $10 \mathrm{~Gb} / \mathrm{s}$ SLR network, which makes it a non-efficient solution to handle high volumes of traffic. Regarding the MLR, despite of presenting a better result in energy efficiency than the $10 \mathrm{~Gb} / \mathrm{s}$, it is also significantly affected by blocking when the traffic load increases. In the high load traffic conditions, the Elastic OFDM network clearly outperforms the other systems, both in energy efficiency and in blocking ratio. This superior performance is justified by its adaptability to different circumstances. In this manner, a low modulation order can be selected to extend the transparent reach (minimizing the regenerations), while a higher one can be used to increase the spectral efficiency, implying a better utilization of the resources. Accordingly, the Elastic OFDMbased can be considered as the most energy efficient solution, outperforming the other options in energy efficiency, and offering the ability of handling higher volumes of traffic. This reduction in the number of required resources is especially significant in such a large network, due to the high number of EDFAs and OXCs that are deployed.

\section{CONCLUSION}

In this paper, the energy efficiency in optical transport networks, considering a dynamic scenario with time-varying connections has been evaluated. The analysis was performed for a flexible-grid OFDM-based network and for a fixed-grid WDM networks with both, a Single Line Rate and a Mixed Line Rate operation. A series of simulations, based on the defined dynamic-routing heuristic algorithms, were carried out in order to check the performance in energy efficiency under different traffic conditions. Furthermore, to check the network size influence, the analysis was applied to two different-sized network topologies. Thus, in a country-sized network, even though the Elastic OFDM network and the MLR network present similar performance in terms of energy efficiency, and slightly better than the $100 \mathrm{~Gb} / \mathrm{s}$, the considerably lower blocking ratio of the Elastic OFDM network makes it the most energy efficient solution, since it is able to handle more traffic with the same resources. The benefits of this solution come from the finer granularity and the use of different modulation formats allowing for a better utilization of the resources, which becomes especially significant under high traffic load. Regarding the continental-sized network, the Elastic OFDMbased is also the most energy efficient solution, showing a better performance than the fixed-grid WDM solutions, not only in terms of network energy efficiency, but also, and specially, in terms of blocking. The benefits, besides the fine granularity and the different modulation formats, come also from the longer transmission reach which allows for reducing the number of the high power consuming regenerations.

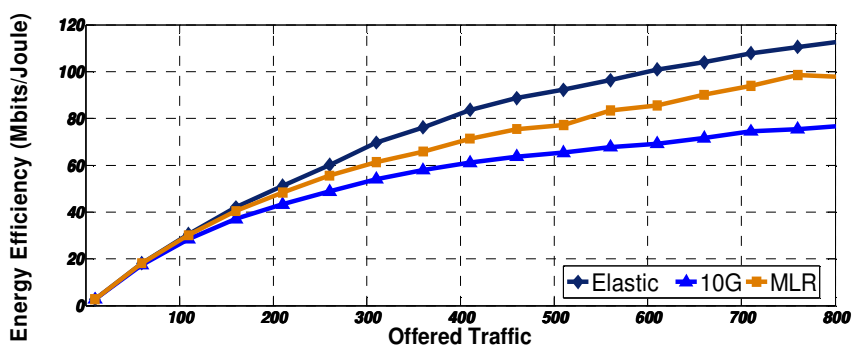

Figure 7. GÉANT2 Network: Energy Efficiency.

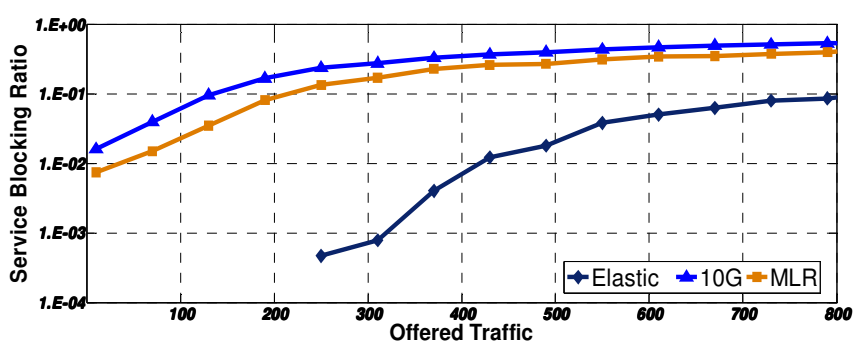

Figure 8. GÉANT2 Network: Service Blocking Ratio.

Based on the results, the Elastic OFDM-based network can be considered as an energy efficient solution for the operation of the future dynamic optical transport networks. This research could be further extended to study the benefits of the sleepmode in the network elements. Besides, an interesting topic for future research is the analysis in terms of energy efficiency of new protection schemes using Elastic OFDM-based network compared to the conventional protection schemes.

\section{ACKNOWLEDGMENT}

The research leading to these results has received funding from the European Community's Seventh Framework Programme [FP7/2007-2013] under grant agreement $\mathrm{n}^{\circ}$ 257740, TREND project, and $n^{\circ} 258644$, CHRON project.

\section{REFERENCES}

[1] CISCO Whitepaper, "Visual Networking Index (VNI)," June 2010.

[2] M. Pickavet, and R. Tucker, "Network Solutions to Reduce the Energy Footprint of ICT," ECOC 2008, Symposium, Sep. 2008.

[3] ITU," ITU and Climate Change Report," Oct. 2008.

[4] M. Jinno, et al., "Demonstration of novel spectrum-efficient elastic optical path network with per-channel variable capacity of $40 \mathrm{~Gb} / \mathrm{s}$ to over 400 Gb/s," ECOC 2008, Sep. 2008.

[5] J. López Vizcaíno, Y. Ye, and I. Tafur Monroy, "Energy Efficiency in Elastic-Bandwidth Optical Networks," International Conference on the Network of The Future, Nov. 2011.

[6] K. Christodoulopoulos, I. Tomkos, and E. Varvarigos, "Dynamic bandwidth allocation in flexible OFDM-based networks," OFC/NFOEC 2011, Paper OTuI5, Mar. 2011.

[7] A. Bocoi, et al., "Cost Comparison of Networks Using Traditional 10 and $40 \mathrm{~Gb} / \mathrm{s}$ Transponders Versus OFDM Transponders," OFC/NFOEC 2008, Paper OThB4, Feb. 2008.

[8] F. Smyth, C. Dorize, and W. van Heddeghem, "GreenTouch Draft Report on Baseline Power Consumption," Version 1.1, April 2011.

[9] J. López Vizcaíno, "Energy Efficiency in Optical Transport Networks," Master's Thesis at Technical University of Denmark (DTU), Sep. 2011

[10] DICONET Project Deliverable, "D.2.1:Definition of dynamic optical network architectures," Version: 1.00, Sep. 2010. 